\title{
Paralympic Judo: Is there Evidence for Match Rigging among Athletes with Disabilities?
}

\author{
${ }^{1}$ Wadim Strielkowski ${ }^{*},{ }^{2}$ Anatoly Shishkin \\ ${ }^{1}$ Cambridge Judge Business School, University of Cambridge, Cambridge, United Kingdom. ${ }^{2}$ Plekhanov Russian \\ University of Economics, Moscow, Russian Federation.
}

\begin{abstract}
Objectives: This paper studies the existence or non-existence of match-fixing (or rigging) among judo wrestlers (judoka) with disabilities during the consecutive Paralympic Games from 1988 until 2016. Methods: In our analysis, we use the institutional framework that makes it easy understand and model the incentives of the wrestlers using the readily available data. Our data set consists of official judo matches that took place during the Paralympic Games from Seoul in 1988 to Rio in 2016. We analyze the distribution of wins across judokas, the medal count at the end of each medal tournaments. Results: Our results are quite similar across specifications. There is no significant evidence to prove that some Paralympic judokas tend to be victorious more often on average than it might be expected. Conclusions: We find no evidence can be found to document match rigging in Paralympic judo wrestling. Our analysis does not confirm the corruption story or rule out effort as the explanation. While the incentive structure of promotion leads to gains from trade between wrestlers on the margin for achieving a winning record and their opponents in some other sports with athletes winning a disproportionate share of the matches when they are on the margin, this does not seem to be the case of Paralympic judo. Reciprocity agreements between Paralympic judo teams from different countries are unlikely to exist, suggesting that collusive behavior is carried out solely by individual actors.
\end{abstract}

KEY WORDS: Judo Wresting, Athletes with Disabilities, Paralympic Games, Match Rigging, Economic Incentives, Regression Analysis, Martial Arts.

\section{INTRODUCTION}

Judo represents a thriving sport with a unique dynamics and special focus on honor, ritual, and history $(1,2)$. Paralympic judo constitutes an interesting stage in its development that is unparalleled in Paralympic movement (3). However, similar to other mainstream sports performed by the normal athletes such as football, ice hockey or athletics, it might become a domain of some irregularities and scandals.
The aim of this paper is not to tackle the recent doping scandals that have shaken the Olympic movement and had many political and social implications (4). Instead, this paper focuses on the issue of match-fixing (or match rigging) or doping scandals notorious in mainstream sports performed by the normal athletes (5).

Match-fixing or match rigging represent a situation when a player (or a group players)

*. Corresponding Author:

Wadim Strielkowski

E-mail: strielkowski@cantab.net 
underperform at a sporting event or contest in order to tilt the outcome with the purpose of achieving the monetary profit or other material or non-material gain (6). The aim of this research is to use the available data in order to demonstrate the existence or non-existence of match rigging in Paralympic sports, namely Paralympic judo.

Paralympic judo as a sport has experienced an unprecedented rise in popularity and participation (7). Since its introduction at the Seoul Paralympic Games with a total of 33 athletes, the participation in London 132 athletes who competed in 15 medal events during the recent Paralympic Games in London in 2012.

Due to the recent popularity of Paralympic Games worldwide, it seems interesting to focus on the role and place of judo in the Paralympic movement, as well as to test its integrity and moral standards. The opportunities for the disabled people to engage themselves in various types of sporting activities are becoming increasingly available thanks to the new developments in both sport disciplines and the new technologies (8).

The Paralympic Games represents a breakthrough sporting event that is a part of the broader Paralympic Movement, which provides a base for people with disabilities to compete in front of the broader audience. Moreover, Paralympic Games also bear a very important message being the representative of the disability rights through integration, equality, opportunity, and non-discrimination (9).

Not only Paralympic judo is a thrilling and noble sport, it also helps millions of disabled people worldwide to overcome the stigma of their diseases and injuries. The most successful male judoka in Paralympic history is Japanese Satoshi Fujimoto (a 3-time winner in the category up to $65 \mathrm{~kg}$ and $66 \mathrm{~kg}$ ). The most successful female judoka was a German Ramona Brussig (who won twice the Paralympics in category under $57 \mathrm{~kg}$ ). Among the most interesting current athletes are Afag Sultanova from Azerbaijan, a former able-bodied judoka who suffered serious injury, but continued competing (she won the category up to $57 \mathrm{~kg}$ at London Paralympics in 2012), or Cuban judoka Hierrezuelo Marcillis (winner of the category up to $90 \mathrm{~kg}$ at the London Paralympic Games in
2012). Taking this all into account it seems very interesting to check whether this noble and positive sport might have indications of match rigging that can unfortunately be found in other disciplines (10).

This paper is structured as follows: Section 2 describes materials and methods focusing on the phenomenon and the world-wide popularity of the Paralympic judo. Section 3 the results of the empirical testing. Section 4 outlines the discussion of the results and their outcomes and possible implications. Finally, section 5 provides overall conclusion for this study.

\section{MATERIALS AND METHODS}

Participants. A qualitative study allows to study the existence or non-existence of match rigging in Paralympic judo, so our selection of the participants was predetermined by this selection. Judo was introduced at the Paralympics in 1988 in Seoul (in the same year, when Olympic Games accepted also the female judo). At Athens 2004 Games women's weight categories were added. At the London 2012 Games there were 15 medal events (attended by 132 athletes). Judo is not a sport for every kind of disability.

Selection and sampling. Only people with visual impairment compete in this sport, thence they matched our selection. Judokas are divided only into weight categories, sight classes B1, B2 and $\mathrm{B} 3$ are left aside (they all compete together). The development in the number of Paralympic judo athletes and the growth in the number of medal events are depicted in Table 1 that follows.

Table 1. Development of Paralympic judo (19882016) (11)

\begin{tabular}{c|c|c|c}
\hline Year & $\begin{array}{c}\text { Paralympic } \\
\text { Games }\end{array}$ & $\begin{array}{c}\text { No. of } \\
\text { athletes }\end{array}$ & $\begin{array}{c}\text { No. of } \\
\text { medal } \\
\text { events }\end{array}$ \\
\hline 1988 & Seoul & 33 & 6 \\
\hline 1992 & Barcelona & 52 & 7 \\
\hline 1996 & Atlanta & 67 & 7 \\
\hline 2000 & Sydney & 83 & 7 \\
\hline 2004 & Athens & 118 & 13 \\
\hline 2008 & Beijing & 129 & 13 \\
\hline 2012 & London & 132 & 15 \\
\hline 2016 & Rio & 129 & 13 \\
\hline
\end{tabular}


Statistical analysis. For the study design, we used the regression analysis approach. Regression analysis represents a linear probability model with standard errors similar to other related match rigging studies (12). We analyze the distribution of wins across Paralympic judokas and the medal count at the end of each medal tournaments looking for the disproportionate number of wrestlers finishing with wins because of the high payoff associated with the win yielding a Paralympic medal. Further evidence comes from estimating regressions of the general form with a wrestlermatch being the basic unit of observation. We include fixed effects for each wrestler and each opponent and specifications coding the wrestleropponent interactions.

\section{RESULTS AND DISCUSSION}

Sample descriptions. Our data set consists of official judo matches that took place during the Paralympic Games from Seoul in 1988 to Rio in 2016, a total of $743(n=743)$ athletes competing in 15 medal categories.

Study design. We look into the cases where the statistical analysis shows irregularities and peculiar cases worth investigating - e.g. when the wins in the match come through too often or when the winner benefits from winning the match more than the losing athlete (e.g. in cases when the medal position are already set).

The literature on the repeated games (socalled "tit-for-tat games") and interactions (13) suggests that the ability to sustain interactions should be positively related to the frequency with which two judo combatants expect to meet in the future since the probability of further meetings implies the existence of more severe punishments for combatants who do not cooperate and tend to cheat.

Empirically, it is possible to approximate for the expected frequency of future combats using two variables: the number of meetings between the two judo combatants that took place in the preceding competitions (in our case previous Paralympic Games), and the fact whether the combatant is in at the end of her or his professional career.
Even though the precise timing of the ending of a judo wrestler's career is not known in advance to the viewers and participants, it is likely that the signals of retirement are apparent (e.g., declining performance, injuries, age, and the like). It takes time to establish a reputation as a good and respected judo combatant who is willing to cooperate and who can be also trusted by her or his opponents, then one might predict that the longer a wrestler has been active in the top ranks of judo, the better she or he will do when she or he is on the declining path of her or his career, and using the similar reasoning, the worse she or he will perform when the opponent is losing. The results of our empirical estimations are reported in the Table 2.

Table 2 reports the percentages of winning the match for wrestlers on the margin on the daily basis day for all days of each Paralympic tournament from 1988 in Seol to 2016 in Rio. We use the linear probability model of any given Paralympic judoka winning a match that is checked for fixed effects that rule out that some matches are included into the dataset twice. The statistical method used in our study is the regression analysis which represents a linear probability model with standard errors similar to other related match rigging studies (12). The variables used in the research are the percentages of winning the match for wrestlers on the margin on the daily basis day. Column (1) in Table 2 shows the results for the judoka's fixed effects. Columns (2) and (3) in Table 2 show the results for the judoka's and her or his opponents' effects.

It is apparent from the results reported in Table 2 above that the predictive power of all three models (denoted hereinafter by (1), (2), and (3)) is very low and does not indicate any results that might yield the significance of the models in question and thence the statistically significant relationship indicating some predictive causality.

Moreover, it is apparent that the coefficients of the respective variables are (in the majority of cases) not significant or have very low significance and predictive power. Our results clearly indicate that there is no apparent statistically significant relationship explaining some forms of fixing or staging the results of the Paralympic judo combats. 
Table 2. Determinants of win likelihood for Paralympic judo wrestlers

\begin{tabular}{|c|c|c|c|c|}
\hline Year & Paralympic Games & (1) & (2) & (3) \\
\hline \multirow{3}{*}{1988} & \multirow{3}{*}{ Seoul } & $0.012(0.002)^{* *}$ & $0.015(0.001)^{*}$ & $0.011(0.001)^{*}$ \\
\hline & & $-0.018(0.007)$ & $-0.007(0.005)$ & $0.016(0.005)^{*}$ \\
\hline & & $-0.015(0.004)$ & $-0.013(0.007)$ & $0.016(0.002)^{* *}$ \\
\hline \multirow{3}{*}{1992} & \multirow{3}{*}{ Barcelona } & $0.018(0.007)^{*}$ & $0.012(0.005)$ & $0.011(0.001)$ \\
\hline & & $0.017(0.006)^{*}$ & $0.014(0.003)^{*}$ & $0.011(0.012)$ \\
\hline & & $0.046(0.006)$ & $0.033(0.006)$ & $0.031(0.003)$ \\
\hline \multirow{3}{*}{1996} & \multirow{3}{*}{ Atlanta } & $0.004(0.008)$ & $0.007(0.005)$ & $0.002(0.002)^{*}$ \\
\hline & & $0.003(0.009)^{* *}$ & $0.011(0.007)$ & $0.001(0.008)^{*}$ \\
\hline & & $0.003(0.008)$ & $0.003(0.006)$ & $0.002(0.006)$ \\
\hline \multirow{3}{*}{2000} & \multirow{3}{*}{ Sydney } & $0.003(0.003)$ & $0.002(0.007)$ & $0.001(0.002)^{*}$ \\
\hline & & $0.001(0.003)$ & $0.003(0.007)$ & $0.004(0.003)^{*}$ \\
\hline & & $0.004(0.003)^{* *}$ & $0.007(0.006)^{*}$ & $0.001(0.002)$ \\
\hline \multirow{3}{*}{2004} & \multirow{3}{*}{ Athens } & $0.007(0.001)$ & $0.006(0.003)$ & $0.011(0.002)$ \\
\hline & & $0.012(0.000)$ & $0.011(0.003)$ & $0.014(0.001)^{*}$ \\
\hline & & $0.006(0.002)$ & $0.005(0.002)$ & $0.011(0.001)$ \\
\hline \multirow{3}{*}{2008} & \multirow{3}{*}{ Beijing } & $0.027(0.004)^{* *}$ & $0.022(0.005)$ & $0.021(0.001)^{*}$ \\
\hline & & $0.093(0.007)$ & $0.072(0.002)$ & $0.003(0.003)$ \\
\hline & & $0.034(0.005)$ & $0.042(0.007)$ & $0.004(0.001)$ \\
\hline \multirow{3}{*}{2012} & \multirow{3}{*}{ London } & $0.015(0.001)$ & $0.015(0.005)$ & $0.005(0.005)$ \\
\hline & & $0.016(0.004)^{*}$ & $0.013(0.002)^{* *}$ & $0.006(0.002)$ \\
\hline & & $0.004(0.001)$ & $0.003(0.006)$ & $0.002(0.002)$ \\
\hline \multirow{3}{*}{2016} & \multirow{3}{*}{ Rio } & $0.019(0.003)^{* *}$ & $0.017(0.005)$ & $0.016(0.001)^{*}$ \\
\hline & & $0.041(0.004)$ & $0.052(0.002)$ & $0.003(0.003)$ \\
\hline & & $0.021(0.004)$ & $0.021(0.007)$ & $0.004(0.002)$ \\
\hline \multicolumn{2}{|r|}{$\mathrm{R}^{2}$} & 0.014 & 0.012 & 0.004 \\
\hline \multicolumn{2}{|r|}{$\mathrm{N}$} & \multicolumn{3}{|c|}{743} \\
\hline
\end{tabular}

Our results yield that the pattern of coefficients does not support the hypothesis about the frequency of match rigging rising as the tournament is coming to an end and the winning combatants might lose a match or two without any significant consequences for their ranking and career in judo. Notably, this gives us grounds to proclaim that the matchfixing in Paralympic judo cannot be verified on the basis of the available empirical data which is quite comforting in comparison with the situation that seems to be taking place in other sports including the Olympic Games (16).

Therefore, our empirical results appear to be consistent with a scenario in which effort is an important determinant of the match outcome and with some judokas having more to gain from a win exerting greater effort and rigor.

\section{CONCLUSION}

Overall, one can conclude that judo represents a truly noble sport that is known for honesty and its deep philosophical roots. Unlike previous studies $(4,5,6)$, it appears unlikely for the judokas to cheat since the material gain from winning is not that big and the concept of cheating goes against the principles of judo. The same can be said about the Paralympic judo that enables disabled athletes to compete at the world top sport events and promote the values of the martial arts and contact sports which is confirmed by the research literature $(8,10,16)$. While the prestige and honor of winning the Paralympic judo tournament might be enormous for the individual wrestlers, the monetary gain and the media coverage cannot be compared to the traditional Olympic games and their tournaments.

Although Paralympic judo does not have very long history and tradition, it can boast 
remarkable results and achievements, making judo a sport for both the professionals and the mainstream public. This makes match rigging at Paralympic judo an issue that might not be worth a risk of taking (9).

The empirical analysis conducted and described in our paper shows that no evidence can be found to document match rigging in Paralympic judo wrestling. Our analysis does not confirm the match rigging or match-fixing at Paralympic judo tournaments or rule out effort as the explanation. While the incentive structure of promotion leads to gains from trade between wrestlers on the margin for achieving a winning record and their opponents in some other sports with athletes winning a disproportionate share of the matches when they are on the margin, this does not seem to be the case of Paralympic judo. Reciprocity agreements between Paralympic judo teams from different countries are unlikely to exist, suggesting that collusive behavior is carried out solely by individual actors.

Based on the results obtained in this paper, we can state that there is no evidence of match rigging amongst athletes with disabilities represented by judokas. This result supports the plausible image of judo as the respected sport with long traditions that attracts honest competitors with solid moral values.

\section{APPLICABLE REMARKS}

- Match-fixing (also known as match rigging) is, alongside with recent doping scandals, one of the most serious issues of modern-day sport. The problem has reached the Olympic movement and the Paralympic Games.

- We study the possibility of matchfixing among judo wrestlers (judoka) with disabilities during the consecutive Paralympic Games from 1988 until 2016 and find no evidence of such behavior.

\section{REFERENCES}

1. Imamura R, Johnson B. Judo. Sports Biomechanics. 2002; 2: 191-201.

2. Miarka B, Del Vecchio F, Julianetti R, Cury R, Camey S, Franchini E. Time-motion and tactical analysis of Olympic judo fighters. International Journal of Performance Analysis in Sport. 2016; 16(1): 133-142.

3. Chang I, Crossman J. When there is a will, there is a way: A quantitative comparison of the newspaper coverage of the 2004 summer Paralympic and Olympic Games. International Journal of Applied Sports Sciences 2009; 2: 16-34.

4. Duval A. The Russian doping scandal at the court of arbitration for sport: lessons for the world anti-doping system. The International Sports Law Journal. 2017; 16(3-4): 177-197.

5. Carpenter K. Match-fixing - The biggest threat to sport in the 21st century? International Sports Law Review. 2012; 2: 13-24.

6. Tak M, Sam M, Jackson S. The politics of countermeasures against match-fixing in sport: A political sociology approach to policy instruments. International Review for the Sociology of Sport, 2016; 1012690216639748

7. Goodger BC, Goodger JM. Judo in the Light of Theory and Sociological Research. International Review for Sociology of Sport. 1977; 2:5-34.

8. Tamari T. Body Image and Prosthetic Aesthetics: Disability, Technology and Paralympic Culture. Body \& Society. 2017; 23(2): 25-56.

9. Pappous A, Hayday E. A case study investigating the impact of the London 2012 Olympic and Paralympic Games on participation in two non-traditional English sports, Judo and Fencing. Leisure studies. 2016; 35(5): 668-684.

10. Bag P, Saha B. Match-Fixing in a Monopoly Betting Market. Journal of Economics \& Management Strategy. 2017; 26(1): 257-289.

11. Paralympic movement. 2017 [2017 August 1] Available at: https://www.paralympic.org/results/historical

12. Dietl, H. M., Lang, M., Werner, S. Corruption in professional Sumo: An update on the study of Duggan and Levitt. Journal of sports economics. 2010; 11(4): 383-396.

13. Fudenberg D, Tirole J. Game theory. Cambridge (MA): MIT Press; 1991. 
14. Gouthon P, Kouassi J, N'Guessan K, Bio-Nigan I, Tonon B, Nouatin B, Akplogan B. Knowledge, Attitude and Practices Regarding Muscular Reinforcement among Judokas in Developing Countries: Case Study of the Republic of Benin. Annals of Applied Sport Science. 2015; 3(2): 11-22.

15. Jahani Golbar S, Gharakhanlou R, Barmaki S, Khazani A, Khorshidi-Hosseini M. A comparison of Age Average of Iranian Medal Winners in Olympic and Asian Games to their Counterparts from Selected Countries. Annals of Applied Sport Science. 2015; 3(4): 69-75.

16. Chappelet J. The Olympic fight against match-fixing. Sport in Society, 2015; 18(10): 1260-1272. 